\title{
The Directed Anti-Oberwolfach Solution: Pancyclic 2-factorizations of complete directed graphs of odd order
}

\author{
Brett Stevens* \\ Department of Mathematics and Statistics \\ Simon Fraser University Burnaby BC V5A 1S6 Canada \\ brett@math.carleton.ca
}

Submitted: September 28, 2000; Accepted: March 26, 2002.

MR Subject Classifications: 05B30, 05C70

\begin{abstract}
The directed anti-Oberwolfach problem asks for a 2-factorization (each factor has in-degree 1 and out-degree 1 for a total degree of two) of $K_{2 n+1}$, not with consistent cycle components in each 2-factor like the Oberwolfach problem, but such that every admissible cycle size appears at least once in some 2 -factor. The solution takes advantage of both Piotrowski's decomposition techniques used to solve Oberwolfach problems and the techniques used by the author to solve the undirected anti-Oberwolfach problem.
\end{abstract}

\section{Introduction}

Suppose that there is a combinatorics conference with $2 n+1$ people attending and it is to be held over $2 n$ days. Each evening there is a dinner which everyone attends. To accommodate the many different sizes of meetings, the conference center has many different sizes of tables. In fact, they have every table size from small two person tables to large round tables seating $2 n+1$ people. When this was noticed, the organizers, having solved the pancyclic seating arrangement the year before [7], asked themselves a harder question: can a seating arrangement could be made for each evening such that every person sits next to every other person exactly once on each side (left and right) over the course of the conference and each size table is used at least once.

Such a schedule, really a decomposition of the complete directed graph, $\vec{K}_{2 n+1}$ into spanning graphs all with in-degree and out-degree both equal one (collections of cycles),

*The author's current address is School of Mathematics and Statistics, Carleton University 1125 Colonel By Dr. Ottawa K1S 5B6 Canada 
would be an example of a 2-factorization of $\vec{K}_{2 n+1}$. Due to their usefulness in solving scheduling problems, 2-factorizations have been well studied. The Oberwolfach problem asks for a 2-factorization in which each subgraph in the decomposition has the same pattern of cycles and much work has been done toward its solution $[1,2,3]$. This corresponds to the conference center using the exact same set of tables each night. Often other graphs besides complete graphs of odd order are investigated. Oberwolfach questions have also been posed and solved for complete bipartite graphs [6]. The problem posed in the introductory paragraph asks that every size cycle appear and so is called the pancyclic 2-factorization problem, or, since it forces such different cycle sizes, the title of 'anti-Oberwolfach problem' emphasizes this contrast.

The conference organizers soon noted, like the year before [7] that tables of size $2 n$, although available, were forbidden since the remaining people would be forced to sit at tables of size 1 which did not exist and would preclude every pair being neighbors on each side exactly once. However, unlike last year tables of size 2 and $2 n-1$ were now permissible due to the directed nature of the problem. After realizing this and doing a preliminary count, the organizers then asked themselves for a schedule that would include the first and last evening with everyone seated around one large table of size $2 n+1$, two evenings with a size 2 table paired with a size $2 n-1$ table, two evenings with a size 3 table paired with a size $2 n-2$ table and so forth up to two evenings with size $n$ table paired with a size $n+1$ table.

If the solution methods from the directed Oberwolfach problem can be paired with methods for the directed anti-Oberwolfach problem, then it is conceivable that that general directed 2-factorization problems can be tackled with great power. This would enable us to answer many different and new scheduling and tournament problems. We have associated our problem with an amusing context but we use it as a convenient showcase to present powerful and very serious construction methods that can contribute to a broader class of 2-factorizations.

The undirected anti-Oberwolfach question has been solved affirmatively for all complete graphs of odd order, complete graphs of even order with a 1-factor removed and all complete bipartite graphs [7]. We show here that all directed complete graphs of odd order have a directed anti-Oberwolfach decomposition. The solution method is a combination of Piotrowski's approach to 2-factorization problems and the methods used to solve the undirected pancyclic 2-factorization. We modify pairs of Hamilton cycles into pairs of 2-factors with the desired cycle structures.

In this paper we offer first some definitions and discussion of directed 2-factorizations, formalizing the notions discussed above. Then we solve the problem. We end with a discussion of the solution method, possible extensions of the problem, and the power these methods provide for constructing very general classes of 2-factorizations.

\section{Definitions and Discussion}

Unless otherwise mentioned, all graphs, and factors are directed. 
Definition 1. A directed pancyclic 2-factorization of a directed graph, $\vec{G}$, of order $v$, is a 2 -factorization of $\vec{G}$ where a cycle of each admissible size, $2,3,4, \ldots, n-3, n-2, n$, appears at least once in some 2 -factor. Such a 2 -factorization is called regular if each cycle size appears equally often.

In this definition, when $n<4$, or $n-2<2$, then the only admissible cycle size is $n$ and the pancyclic 2-factorizations in these cases are trivial.

With this definition in mind, the directed anti-Oberwolfach problem asks for a regular pancyclic 2-factorization of $\vec{K}_{2 n+1}$. In every case, counting shows that the all the 2-factors, except the two Hamilton 2-factors in $\vec{K}_{2 n+1}$ must be of the form: an $i$-cycle and a $v-i$ cycle. We define here a notation to refer to the different possible kinds of 2-factors:

Definition 2. An $i,(v-i)$-factor is a 2-factor of an order $v$ graph, $\vec{G}$, that is the disjoint union of an $i$-cycle and a $(v-i)$-cycle.

To produce 2-factors with the desired cycle structures we will decompose the union of either two or four Hamilton 2-factors into 2-factors with smaller cycles. These decompositions will be achieved by taking the union to two Hamilton factors from a Walecki decomposition of $\vec{K}_{2 n+1}$, either consecutive pairs as used in [7] or orthogonal pairs, as used by Piotrowski [5]. In [7] these methods of decomposition were fully formalized and for formal structure we refer the reader there.

In each case the solution method will be similar. We will present a Hamilton 2factorization of each graph in question where the 2-factors are cyclic developments of each other. We will then decompose the union of either consecutive or orthogonal pairs or quadruples of Hamilton 2-factors into 2-factors with the desired cycle structures. The fact that the set of Hamilton factors are cyclically generated from each other guarantee that any union of two consecutive Hamilton factors are isomorphic to the union of any other consecutive pairs and similarly for the pairs of orthogonal Hamilton factors. Thus we can formulate general statements about the possible decompositions of these unions.

\section{Solution for $\vec{K}_{2 n+1}$}

We will have to consider the the cases where $n \equiv 0 \bmod 2$ and $n \equiv 1 \bmod 2$ separately because we will need to use slightly different Hamilton decompositions. These correspond, respectively to the cases $v \equiv 1 \bmod 4$ and $v \equiv 3 \bmod 4$.

\section{$3.1 v \equiv 1 \bmod 4$}

For the case $v=4 u+1$, we construct 2-factors by using the directed analogue of the Walecki 2-factorization of $\vec{K}_{4 u+1}$ given by Lucas [4]. Let the vertices of $\vec{K}_{4 u+1}$ be represented by the set $\mathbb{Z}_{4 u} \cup\{\infty\}$. Then the first 2 -factor, $F_{u}$, is the directed cycle

$(\infty 014 u-124 u-33 \ldots 2 u+12 u)$. 


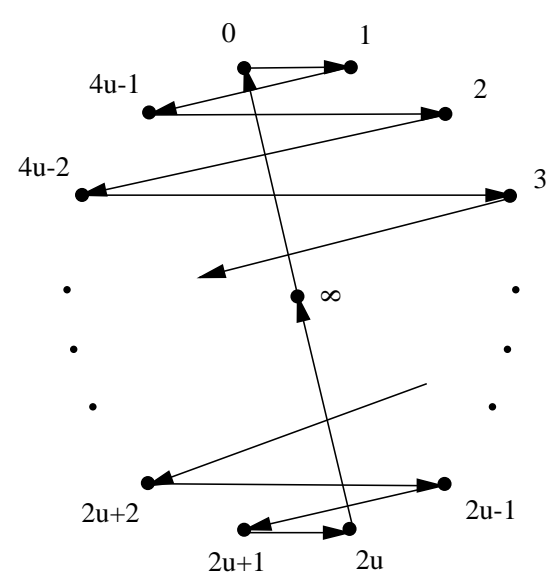

Figure 1: A standard directed Hamilton 2-factor of $\vec{K}_{4 u+1}$.

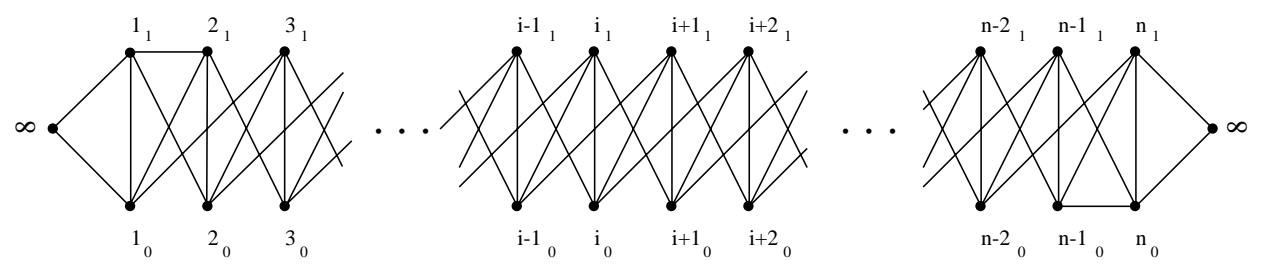

Figure 2: The union of two consecutive undirected 2-factors, $F_{u} \cup \sigma\left(F_{u}\right)$.

This 2-factor is shown in Figure 1. All other 2-factors are developed from $F_{u}$ by application of the cyclic automorphism $\sigma$ where $\sigma(\infty)=\infty, \sigma(i)=i+1(i<4 u-1)$ and $\sigma(4 u-1)=0$. The map $\sigma$ can be viewed as clockwise rotation of the first 2-factor shown in Figure 1. Its action on the set of 2 -factors is order $4 u$.

Lemma 3. The union of $\sigma^{i}\left(F_{u}\right), \sigma^{i+1}\left(F_{u}\right), \sigma^{i+2 u}\left(F_{u}\right)$ and $\sigma^{i+2 u+1}\left(F_{u}\right)$ can be decomposed into four 2-factors such that the first two are $2 i+1, v-2 i-1$-factors and the second two are $2 j+1, v-2 j-1$-factors, for any $1 \leq i \neq j \leq 2 u-2$. Or alternatively the first two are $2 i+1, v-2 i-1$-factors and the second two are directed Hamilton cycles, for any $1 \leq i \leq 2 u-2$.

Proof. It is proved in [7] that the union of any two consecutive undirected Walecki Hamilton 2-factors, shown in Figure 2, can be decomposed into two 2-factors where the first is a $2 i+1, v-2 i-1$-factor and the second is a $2 j+1, v-2 j-1$-factor, for any $1 \leq i \neq j \leq 2 u-2$ or the first is a $2 i+1, v-2 i-1$-factor and the second is a Hamilton cycle. The four directed Hamilton 2-factors in the hypothesis of the lemma contain an arc in both directions wherever there is an arc in the union of any two consecutive undirected Walecki 2 -factors. Hence by doubling and oppositely directing the cycles constructed in [7] we construct the desired four directed 2-factors.

Lemma 4. The union of $\sigma^{\ell}\left(F_{u}\right)$ and $\sigma^{\ell+u}\left(F_{u}\right)$ is isomorphic to the graph shown in Figure 3 and can be decomposed into a $2 j+1, v-2 j-1$-factor and a $2 j+2, v-2 j-2$-factor 


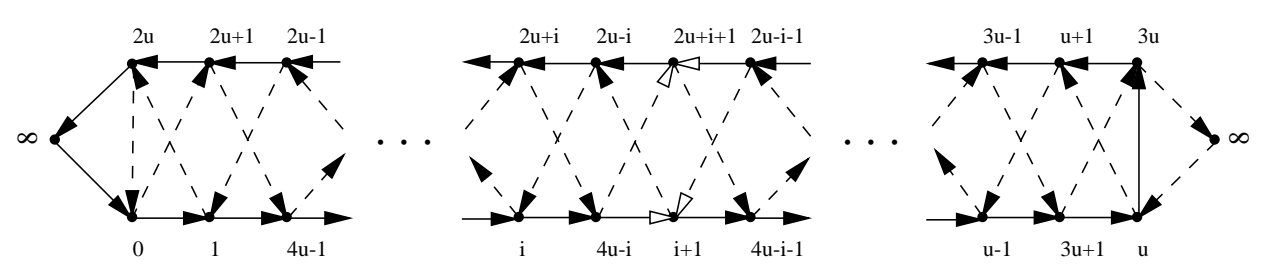

Figure 3: The union of $F_{u}$ and $\sigma^{u}\left(F_{u}\right)$. Note $i \leq u$.
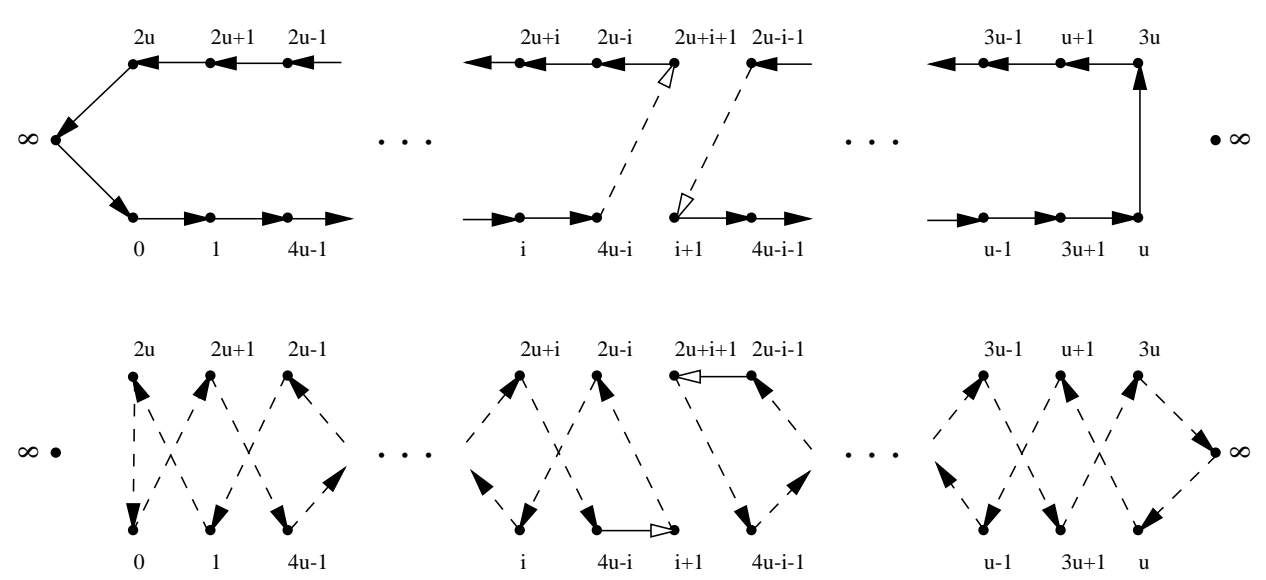

Figure 4: A $4 i+4, v-4 i-4$-factor and a $4 i+3, v-4 i-3$-factor produced from $F_{u} \cup \sigma^{u}\left(F_{u}\right)$.

where $1 \leq j \leq 2 u-2$.

Proof. Because the $F_{u}$ are developed cyclically we can assume that $\ell=0$. In Figure 3, the solid arcs are arcs from $F_{u}$ and the dashed arcs are from $\sigma^{u}\left(F_{u}\right)$. Switching the pairs with the hollow arrow heads produces a $4 i+4, v-4 i-4$-factor and a $4 i+3, v-4 i-3$-factor. As $0 \leq i \leq u-2$ and the order of the 2 -factors and the order of the cycles within each two factor may be switched, we can construct all the needed 2-factors. The resulting directed 2-factors are shown in Figure 4.

Lemma 5. For $u \geq 2$, the union of $\sigma^{\ell}\left(F_{u}\right), \sigma^{\ell+u}\left(F_{u}\right), \sigma^{\ell+2 u}\left(F_{u}\right)$ and $\sigma^{\ell+3 u}\left(F_{u}\right)$ can be decomposed into two 2, $v-2$-factors and two 3,v-3-factors.

Proof. Without loss of generality, $\ell=0$ and the union of the four graphs is the same as the graph shown in Figure 3 except that it contains an arc in each direction where there is any arc. It can be decomposed, in the manner shown in Figure 5, into two 2, $v-2$-factors and two $3, v-2$-factors.

Lemma 6. There is no regular pancyclic directed 2-factorization of $\vec{K}_{5}$.

Proof. Such a decomposition would consist of two 2, 3-factors and two Hamilton 2-factors. It is easy to check that if the two digons are vertex disjoint then each must duplicate an 

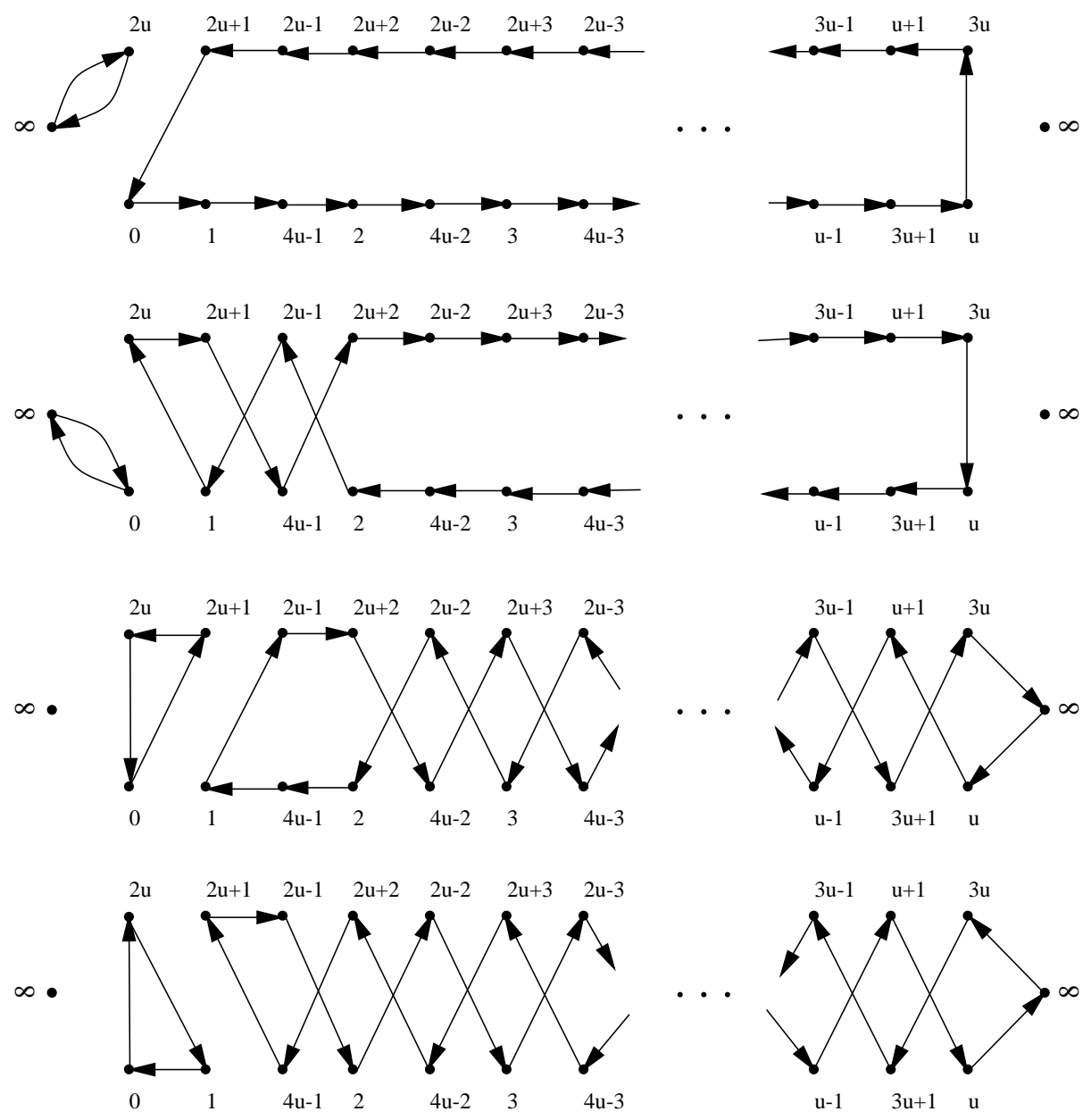

Figure 5: The decomposition of $F_{u} \cup \sigma^{u}\left(F_{u}\right) \cup \sigma^{2 u}\left(F_{u}\right) \cup \sigma^{3 u}\left(F_{u}\right)$ into two 2, $v$ - 2-factors and two $3, v-3$-factors. 


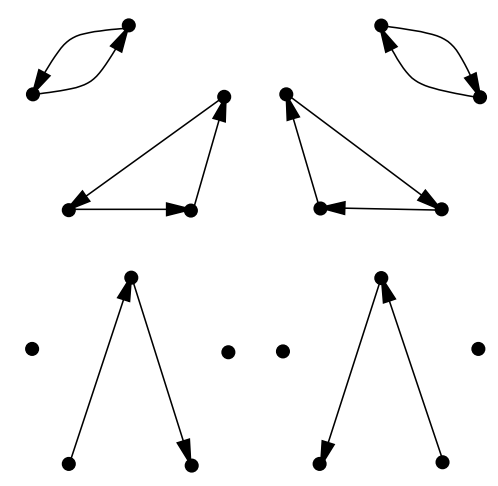

Figure 6: Incomplete pancyclic directed 2-factorization of $\vec{K}_{5}$.

arc that is in the triangle corresponding to the other digon. This shows that the two digons must intersect in exactly one vertex. The picture is shown in Figure 6. It is easily verified that it is impossible to fill in the rest of the arcs without conflict.

Theorem 7. $\vec{K}_{4 u+1}$ can be regularly pancyclically decomposed if and only if $u \geq 2$.

Proof. There is no pancyclic direct 2-factorization of $\vec{K}_{5}$. When $u=2$ the following cycles are a pancyclic directed 2-factorization of $\vec{K}_{9}$ :

( 0122345678 )

( 021354687 )

$(03)(1427586)$

$(041)(257638)$

$(0516)(24837)$

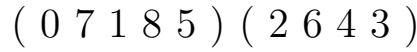

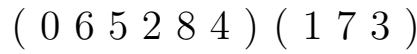

$(0815362)(47)$

For $u \geq 3$, apply Lemma 5 to $F_{u} \cup \sigma^{u}\left(F_{u}\right) \cup \sigma^{2 u}\left(F_{u}\right) \cup \sigma^{3 u}\left(F_{u}\right)$ to construct two $2, v-2$ factors and two 3, $v-3$-factors. Apply Lemma 3 to $\sigma\left(F_{u}\right) \cup \sigma^{2}\left(F_{u}\right) \cup \sigma^{1+2 u}\left(F_{u}\right) \cup \sigma^{2+2 u}\left(F_{u}\right)$ to construct a two Hamilton 2-factors and two 4,v-4-factors. And again apply Lemma 3 to $\sigma^{1+u}\left(F_{u}\right) \cup \sigma^{2+u}\left(F_{u}\right) \cup \sigma^{1+3 u}\left(F_{u}\right) \cup \sigma^{2+3 u}\left(F_{u}\right)$ to construct two 5, $v-5$-factors and two $6, v-6$-factors. When $u>4$, for $3 \leq i \leq u-1$ apply Lemma 4 to $\sigma^{i}\left(F_{u}\right) \cup \sigma^{i+u}\left(F_{u}\right)$ and to $\sigma^{i+2 u}\left(F_{u}\right) \cup \sigma^{i+3 u}\left(F_{u}\right)$ to construct two $2 i+1, v-2 i-1$-factors and two $2 i+2, v-2 i-2$ factors.

\section{$3.2 v \equiv 3 \bmod 4$}

To tackle the case when $v=4 u+3$ we use a different Hamilton factorization. Instead of having one "infinite" vertex as in Subsection 3.1 we have three, $\infty, \infty_{1}$, and $\infty_{-1}$. The subscripts 1 and -1 refer to the similar role to the arcs of length 1 and -1 that these two "infinite" points play. Let the vertices of $\vec{K}_{4 u+3}$ be represented by the set $\mathbb{Z}_{4 u} \cup\left\{\infty, \infty_{1}, \infty_{-1}\right\}$. Then the first 2-factor, $F_{u}$, is the directed cycle 


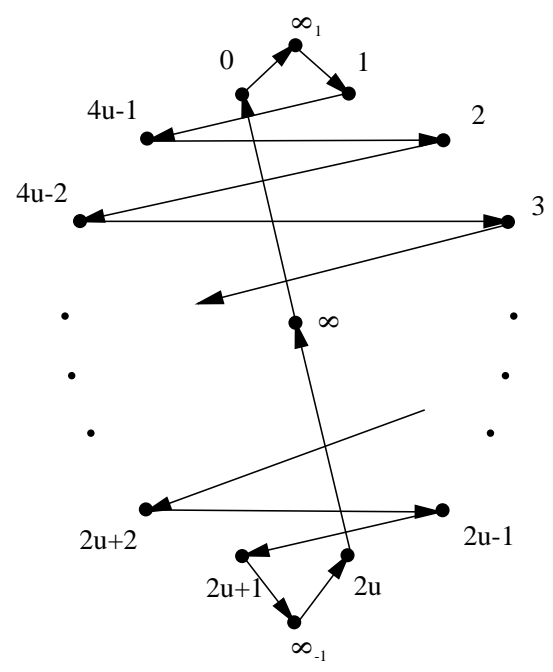

Figure 7: A standard directed Hamilton 2-factor of $\vec{K}_{4 u+3}$.

$\left(\infty 0 \infty_{1} 14 u-124 u-33 \ldots 2 u+1 \infty_{-1} 2 u\right)$.

This 2-factor is shown in Figure 7. $4 u-1$ other 2-factors are developed from $F_{u}$ by application of the cyclic automorphism $\sigma$ where $\sigma(\infty)=\infty, \sigma\left(\infty_{1}\right)=\infty_{1}, \sigma\left(\infty_{-1}\right)=$ $\infty_{-1}, \sigma(i)=i+1(i<4 u-1)$ and $\sigma(4 u-1)=0$. The map $\sigma$ can be viewed as clockwise rotation of the first 2-factor shown in Figure 7 . Its action on the set of 2 -factors is order $4 u$. The last two 2-factors are the factor generated by all arcs of length 1 and the cycle $\left\{\infty, \infty_{1}, \infty_{-1}\right\}$ and the reverse orientation of this factor. These are shown in Figure 8 . These final two 2-factors will remain as they are being used directly as the two 3, $v-3$ factors that we need. The rest of the needed 2-factors will be constructed from the unions of pairs, $\sigma^{\ell}\left(F_{u}\right) \cup \sigma^{\ell+u}\left(F_{u}\right)$ or quadruples $\sigma^{\ell}\left(F_{u}\right) \cup \sigma^{\ell+u}\left(F_{u}\right) \cup \sigma^{\ell+2 u}\left(F_{u}\right) \cup \sigma^{\ell+3 u}\left(F_{u}\right)$ of the cyclically developed Hamilton 2-factors by the following lemmas. Because the Hamilton 2 -factors are developed cyclically we can assume that $\ell=0$ and the same considerations discussed in Subsection 3.1 show us that $F_{u} \cup \sigma^{u}\left(F_{u}\right)$ is isomorphic to the graph shown in Figure 9. The solid lines are the arcs from $F_{u}$ and the dashed lines are the arcs from $\sigma^{u}\left(F_{u}\right)$. Similarly $F_{u} \cup \sigma^{u}\left(F_{u}\right) \cup \sigma^{2 u}\left(F_{u}\right) \cup \sigma^{3 u}\left(F_{u}\right)$ is isomorphic to the graph shown in Figure 10. The solid lines are the arcs from $F_{u}$ and $\sigma^{2 u}\left(F_{u}\right)$ and the dashed lines are the arcs from $\sigma^{u}\left(F_{u}\right)$ and $\sigma^{3 u}\left(F_{u}\right)$.

A nearly identical construction to that of Lemma 4 gives the following lemma. This lemma is not used in the specific problem solved herein but maybe useful for other constructions.

Lemma 8. $F_{u} \cup \sigma^{u}\left(F_{u}\right)$ can be decomposed into $a+4 i, v-6-4 i$-factor and a $3+4 i, v-$ $3-4 i$-factor for all $1 \leq i \leq \frac{v-11}{4}$.

Lemma 9. $F_{u} \cup \sigma^{u}\left(F_{u}\right) \cup \sigma^{2 u}\left(F_{u}\right) \cup \sigma^{3 u}\left(F_{u}\right)$ can be decomposed into two 2, $v-2$-factors and two Hamilton 2-factors. 

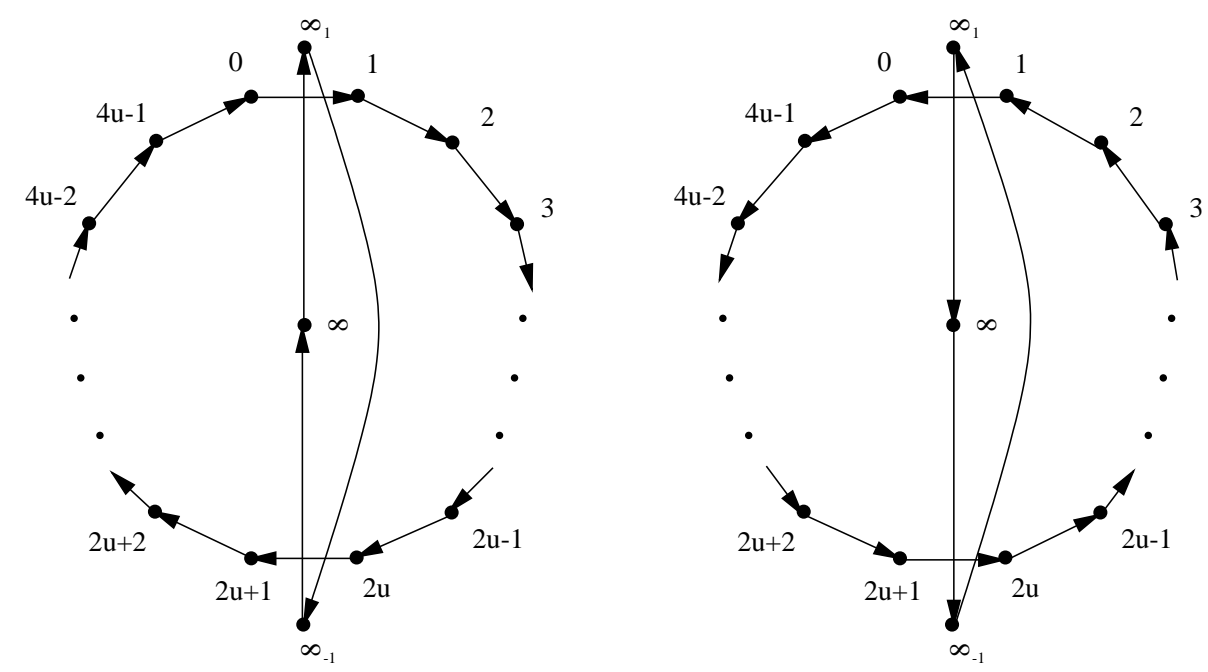

Figure 8: the final two 2-factors of $\vec{K}_{4 u+3}$.
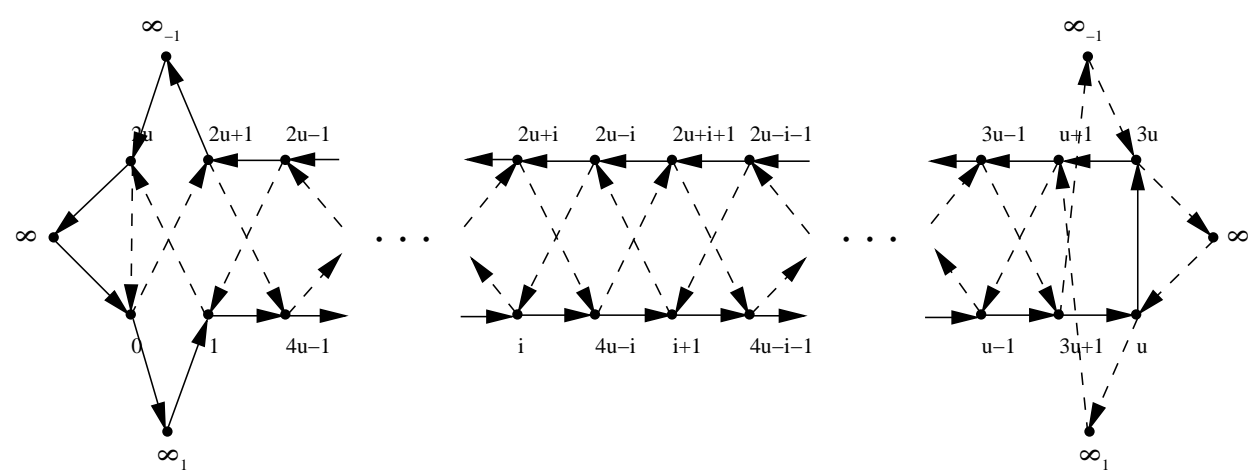

Figure 9: $F_{u} \cup \sigma^{u}\left(F_{u}\right)$.
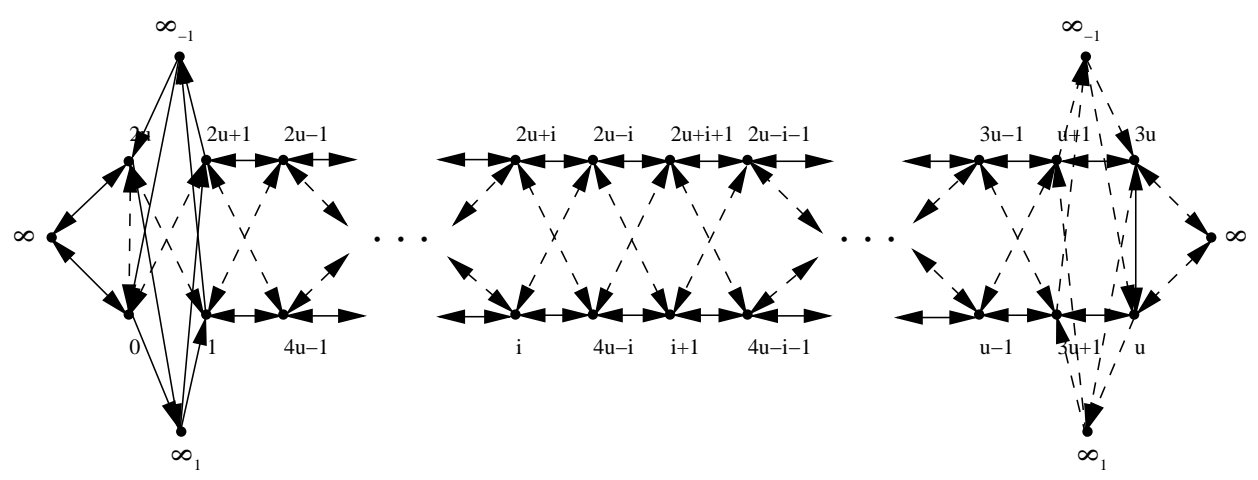

Figure 10: $F_{u} \cup \sigma^{u}\left(F_{u}\right) \cup \sigma^{2 u}\left(F_{u}\right) \cup \sigma^{3 u}\left(F_{u}\right)$. 

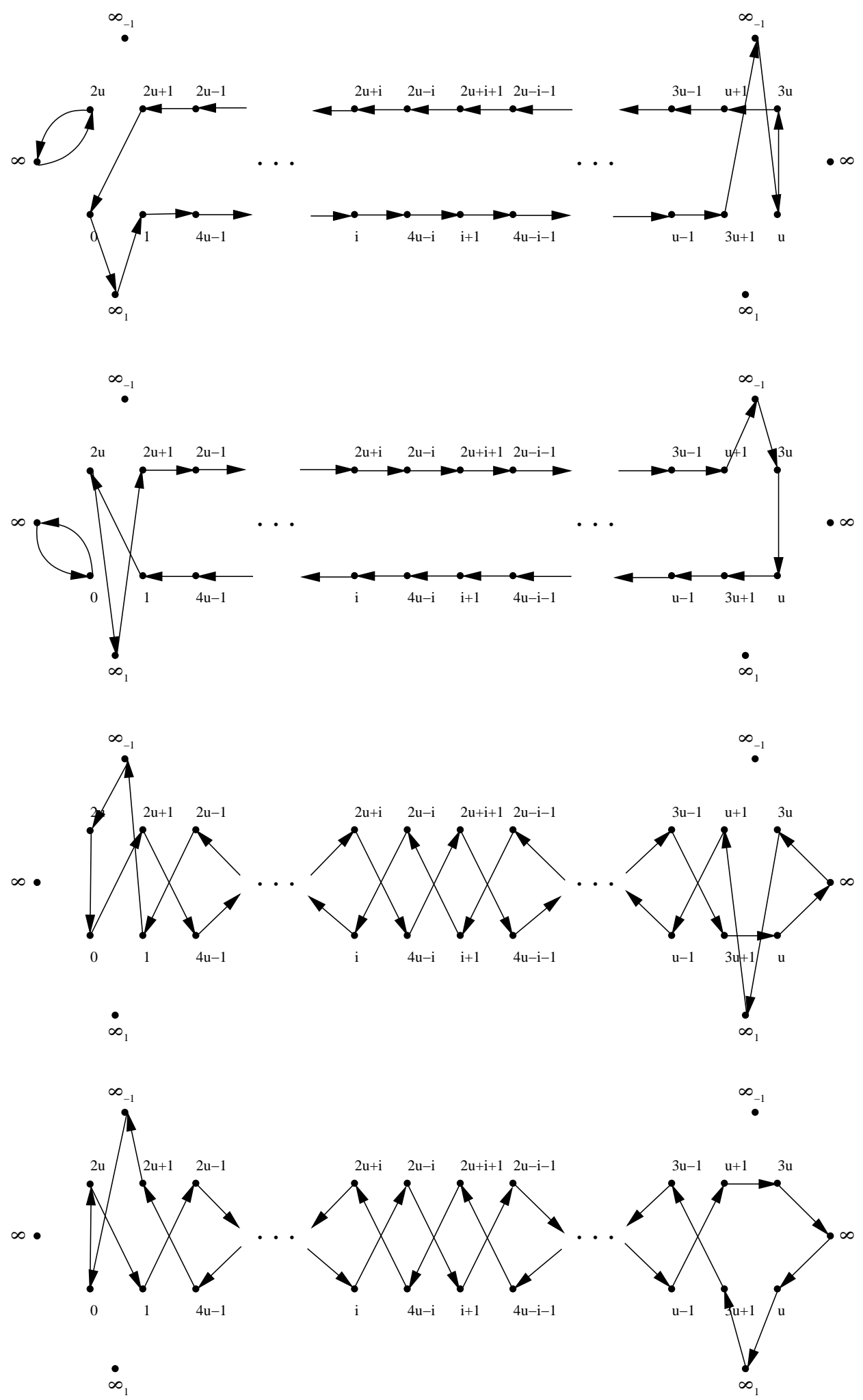

Figure 11: Decomposition of $F_{u} \cup \sigma^{u}\left(F_{u}\right) \cup \sigma^{2 u}\left(F_{u}\right) \cup \sigma^{3 u}\left(F_{u}\right)$ into two 2, $v$-2-factors and two Hamilton 2-factors. 
Proof. Figure 11 shows the decomposition.

Lemma 10. $F_{u} \cup \sigma^{u}\left(F_{u}\right) \cup \sigma^{2 u}\left(F_{u}\right) \cup \sigma^{3 u}\left(F_{u}\right)$ can be decomposed into two 4,v-4-factors and two 5,v-5-factors.

Proof. Figure 12 shows the decomposition.

Lemma 11. $F_{u} \cup \sigma^{u}\left(F_{u}\right) \cup \sigma^{2 u}\left(F_{u}\right) \cup \sigma^{3 u}\left(F_{u}\right)$ can be decomposed into a $4 i+4, v-4 i-4$ factor a $4 i+5, v-4 i-5$-factor, a $4 j+4, v-4 j-4$-factor and a $4 j+5, v-4 j-5$-factor for $1 \leq i, j \leq \frac{v-11}{4}$.

Proof. Figure 13 shows the decomposition.

Theorem 12. There exists a pancyclic 2-factorization of $\vec{K}_{4 u+3}$ for all $u \geq 0$.

Proof. The pancyclic 2-factorization of $\vec{K}_{3}$ consists of two Hamilton cycles and is trivially found. A pancyclic 2-factorization of $\vec{K}_{7}$ is

$\left(\begin{array}{lllllll}0 & 1 & 2 & 3 & 4 & 5 & 6\end{array}\right)$

$\left(\begin{array}{lllllll}0 & 2 & 1 & 3 & 6 & 5 & 4\end{array}\right)$

(0 5) $\left(\begin{array}{lllll}1 & 4 & 2 & 6 & 3\end{array}\right)$

$\left(\begin{array}{lll}1 & 6 & 4\end{array}\right)\left(\begin{array}{llll}0 & 3 & 5 & 2\end{array}\right)$

$\left(\begin{array}{llll}0 & 4 & 6 & 1\end{array}\right)\left(\begin{array}{lll}2 & 5 & 3\end{array}\right)$

$\left(\begin{array}{lllll}0 & 6 & 2 & 4 & 3\end{array}\right)\left(\begin{array}{ll}1 & 5\end{array}\right)$

A pancyclic 2-factorization of $\vec{K}_{11}$ is

$\left(\begin{array}{llllllll}0 & 1 & 2 & 345678 & 5 & 1011 & 121314\end{array}\right)$

$\left(\begin{array}{llllllll}0 & 2 & 135468711 & 10121413\end{array}\right)$

(0 3) (1425 4699810131114 12)

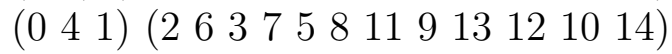

$(0516)(2438127109141113)$

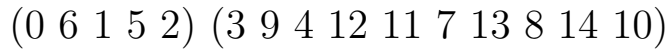

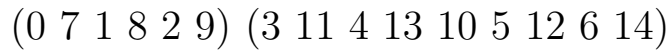

$\left(\begin{array}{llllll}0 & 8 & 1 & 7 & 210 & 4\end{array}\right)(313611514912)$

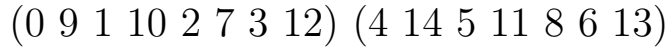

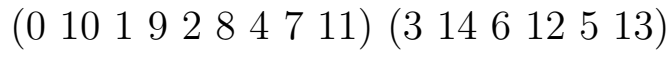

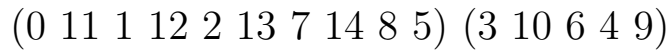

$(01248139536107)(111214)$

$(0135971283114410)(2116)$

(0147411321296510 8) (1 13)

For $u \geq 3$ we form the 2-factors that contain digons and two Hamilton 2-factors using Lemma 9 from $F_{u} \cup \sigma^{u}\left(F_{u}\right) \cup \sigma^{2 u}\left(F_{u}\right) \cup \sigma^{3 u}\left(F_{u}\right)$. Construct the 4, $v-4$-factors and 5, $v-5$ factors from $\sigma\left(F_{u}\right) \cup \sigma^{1+u}\left(F_{u}\right) \cup \sigma^{1+2 u}\left(F_{u}\right) \cup \sigma^{1+3 u}\left(F_{u}\right)$ using Lemma 10. For $0 \leq i \leq u-3$ construct two $6+2 i, v-6-2 i$-factors and two $7+2 i, v-7-2 i$-factors using Lemma 11 . All these two factors and the two 3,v-3-factors shown in Figure 8 are a pancyclic 2-factorization of $\vec{K}_{4 u+3}$. 

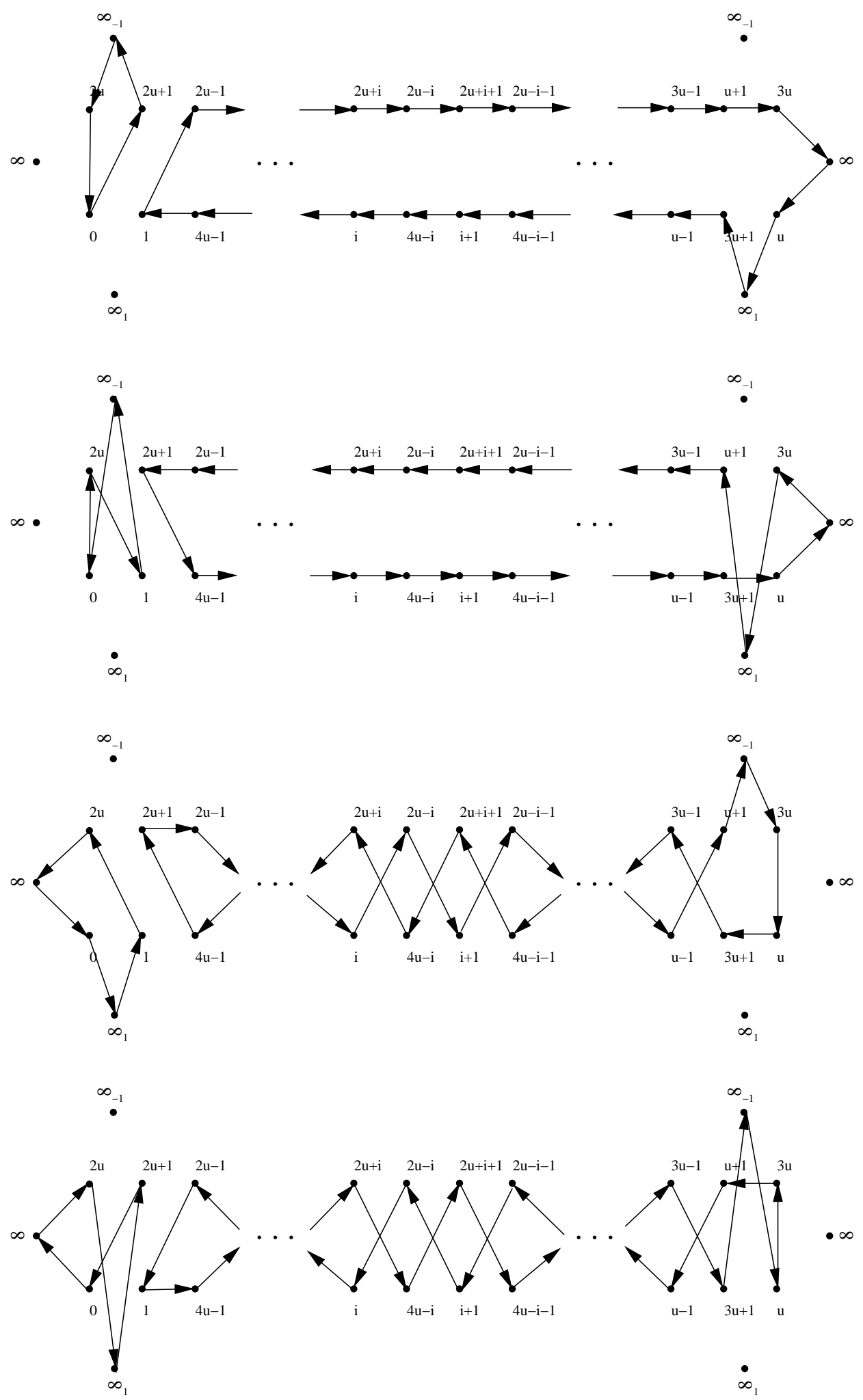

Figure 12: Decomposition of $F_{u} \cup \sigma^{u}\left(F_{u}\right) \cup \sigma^{2 u}\left(F_{u}\right) \cup \sigma^{3 u}\left(F_{u}\right)$ into two 4, $v$-4-factors and two $5, v-5$-factors. 

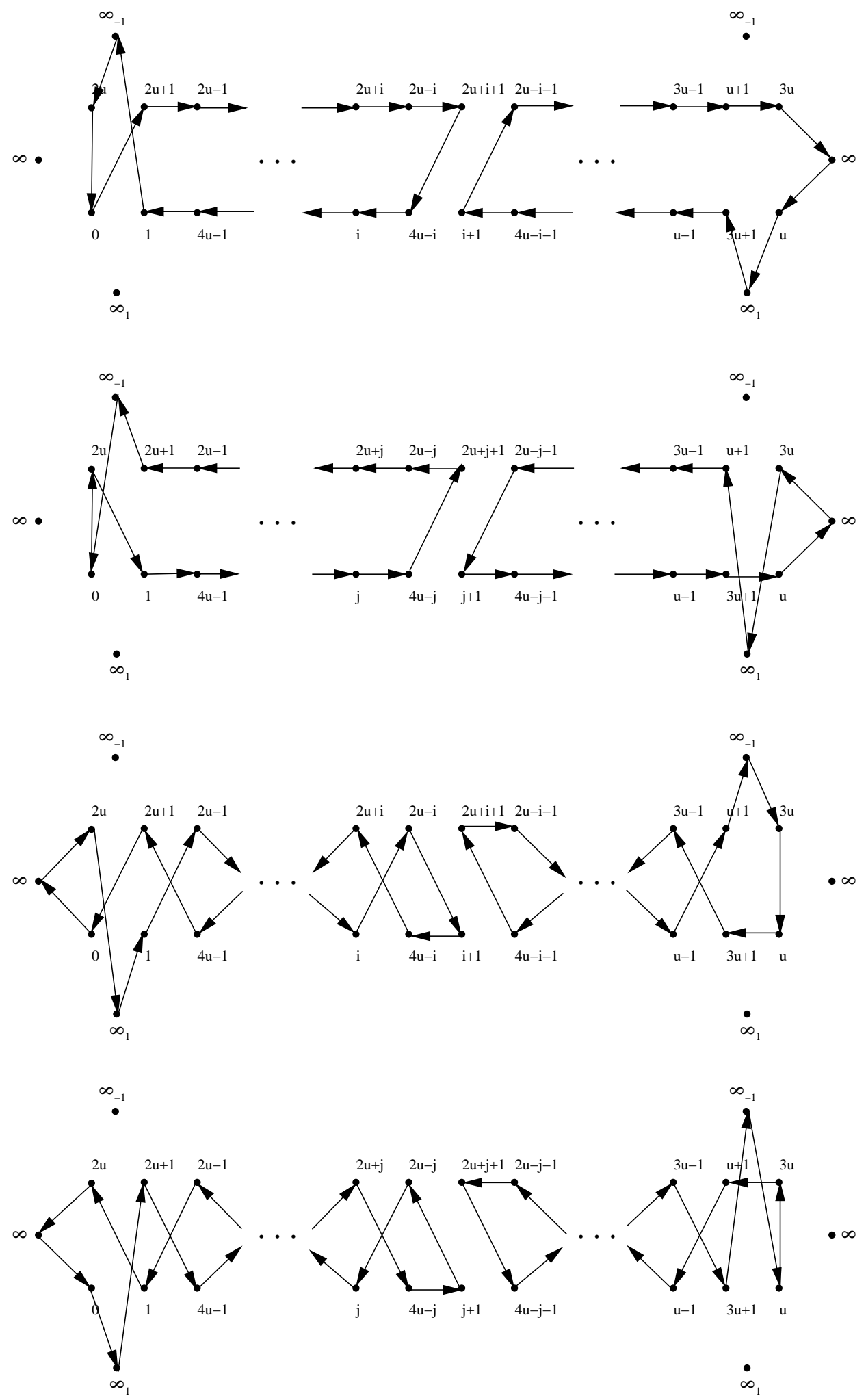

Figure 13: Decomposition of $F_{u} \cup \sigma^{u}\left(F_{u}\right) \cup \sigma^{2 u}\left(F_{u}\right) \cup \sigma^{3 u}\left(F_{u}\right)$ into a $4 i+4, v-4 i-4$-factor a $4 i+5, v-4 i-5$-factor, a $4 j+4, v-4 j-4$-factor and a $4 j+5, v-4 j-5$-factor. 
We did not use Lemma 8 but we include it to state the full flexibility of possible cycle decompositions available to us. Most previous 2-factorization problems ask for uniform 2 -factors or only allow a small range of cycle sizes. We have used the recreational antiOberwolfach problem to introduce the study of admitting a wider range of cycles sizes and less uniform 2-factors. Solving it is an example of the power of the decomposition lemmas.

\section{Conclusion}

As a demonstration of a powerful method for a wide range of directed 2-factorization problems we have solved the directed pancyclic 2-factorization for all directed complete graphs of odd order except $\vec{K}_{5}$ which we prove has no directed pancyclic 2-factorization. We start with a Walecki or slight modification of a Walecki 2-factorization of the complete graph in question with a cyclic automorphism group. The union of consecutive or orthogonal pairs of the 2-factors is shown to be decomposable into two 2-factors with a wide range of cycle structures by judicious swapping of edges between decompositions of these unions. This flexibility of decomposition and the automorphism group allow the desired solution to be constructed. The use of these methods to solve the pancyclic 2 -factorization problem indicates the strength and range of the constructions.

We propose that the methods outlined in this article might be useful for the following problem: The construction of directed 2-factorizations with prescribed lists of cycle types for each 2-factor. If each element in the list is a 2-factor with one or two cycles, then the methods presented here nearly complete the problem. The only obstacle towards the solution of these problems is the construction of pairs of 2-factors with the same cycle type, the Oberwolfach aspect of the question.

The obvious next cases to solve are complete even directed graphs, $\vec{K}_{2 n}$ and complete

directed bipartite graphs, $\vec{K}_{n, n}$. We have begun work on these and have found them more challenging than case of the directed complete graph of odd order, $\vec{K}_{2 n+1}$. The primary difficulty seems to be to construct the two required 2 -factors that contain digons. We believe that the answer to both these questions is affirmative and that similar methods to those used here will achieve the result.

\section{Acknowledgments}

I would like to thank Prof. E. Mendelsohn and Prof. A. Rosa for initially proposing the anti-Oberwolfach family of problems. The author was supported by PIMS postdoctoral fellowship, the Department of Mathematics and Statistics at Simon Fraser University, MITACS and IBM Watson Research. 


\section{References}

[1] B. Alspach and R. Häggkvist. Some observations on the Oberwolfach problem. Journal of Graph Theory, 9:177-187, 1985.

[2] B. Alspach, P. Schellenberg, D. Stinson, and D. Wagner. The Oberwolfach problem and factors of uniform odd length cycles. J. Combin. Theory. Ser. A, 52:20-43, 1989.

[3] C. J. Colbourn and J. H. Dinitz, editors. The CRC Handbook of Combinatorial Designs. CRC Press, Boca Raton, 1996.

[4] E. Lucas. Récréations Mathématiques, volume 2. Gauthier-Villars, Paris, 1883.

[5] W. Piotrowski. Untersuchungen über das Oberwolfacher Problem. Arbeitspapier, 1979.

[6] W. Piotrowski. The solution of the bipartite analogue of the Oberwolfach problem. Discrete Math., 97:339-356, 1991.

[7] B. Stevens. The anti-Oberwolfach solution: Pancyclic 2-factorizations of complete graphs. Theoret. Comput. Sci. (To Appear). 